\title{
NOTE ON THE BACTERIOLOGY OF BRONCHO-PNEUMONIA OF CHILDREN.
}

\author{
BY \\ W. (GLEN LISTON, C.I.E., M.D., I.M.S. (retd.) \\ Bacteriologist, Royal College of Physicians, Edinburgh.
}

Invited by Drs. McNeil, Macgregor and Alexander to co-operate with them in the study of broncho-pneumonia in clildren and to investigate the bacteriological cause of this very fatal malady, I was summoned to attend the first post-mortem examination of their series on the 25th February, 1926. Two fatal cases of broncho-pneumonia (see Appendix, Cases 1 and 2) were examined on that day. The naked-eye post-mortem appearances in both cases reminded me of that type of fatal broncho-pneumonia which occurs in certain cases of influenza, and of which I had seen many examples during the terrible epidemic of 1917-18 in Bombay and India. The work then carried through in cooperation with my colleagues at the Bombay Bacteriological Laboratory, had shown that, while it was comparatively easy to demonstrate the presence of Pfeiffer's bacillus by culture when a special medium was used, it was much more difficult to do so when the ordinary laboratory media were employed, including in this category blood agar.

To the post-mortem examinations above mentioned I had brought with me for use a variety of the culture media generally employed in routine laboratory investigations, (for example, agar, serum agar, and blood agar), but I had not brought with me any special medium essential for the study of influenza, as I had not imagined that I might be called upon to study a complication of that disease. For these two first examinations, therefore, I had to make use of such media as were at hand, but in addition to cultures from the lungs and sputum, I prepared smears from the sputum and inflamed lung. These smears were examined after staining by Gram's method; they revealed the presence of large numbers of minute Gram-negative bacilli having the morphological characters of Pfeiffer's organism. The organisms were more abundant in the sputum of both cases, but they were also demonstrated in the smears from the lungs. In the first of the two cases, although Pfeiffer's bacillus preponderated, other organisms were also present, but in the lung smears only Pfeiffer's bacillus could be seen. Pfeiffer's bacillus was also isolated from a culture made from the lung in Case No. 2.

\section{Technique Employed.}

In all subsequent examinations a special medium, Soparkar's, was used for cultivations. This medium has been found to be very suitable for the detection of Pfeiffer's bacillus. The method of preparing the medium has been described in the Indian Journal of Medical Research ${ }^{1}$. In addition to the fact that Pfeiffer's bacillus grows well on this medium it has this advantage over many of the other media used for the cultivation of that organism : it is transparent, so that it is possible to study the characters of the colonies which develop upon it by transmitted as well as reflected light. This is a matter of considerable moment in distinguishing colonies of Pfeiffer's bacillus from other colonies simulating it. 
A routine system of examination was carried through in all cases where this was possible. After the chest had been opened and the pericardium incised, the surface of the heart was seared with a hot iron, and by means of a sterile capillary pipette and teat, passed through the sterilized surface of the heart, a sample of the blood in the heart was withdrawn and spread on the surface of a Soparkar agar slope. The lungs were then removed from the chest, a consolidated area was selected, the surface of the pleura over this area was seared and a capillary pipette with teat attached was passed through the seared pleura into the consolidated tissue immediately below the surface of the pleura, and by manipulating the teat and capillary tube a small quantity of blood-stained fluid was obtained. A small drop of this fluid was placed in the centre of a Petri dish filled with Soparkar's medium, and with a sterile glass rod, bent at a right angle, the drop of fluid was spread over the surface of the plate. Such cultures may be called for convenience lung-puncture cultures. In a similar manner a plate was prepared from the sputum which was usually withdrawn from the trachea by means of a capillary pipette. These may be designated sputum cultures. Slides for microscopical examination were prepared at the same time. In almost all the cases the lymphatic glands at the hilum of the lungs were much enlarged and inflamed, but it was only in three cases at the end of the series that cultures were made from this source. Where there was an excess of fluid or pus in the pleura, pericardium, or peritoneum, cultures were made from the fluid, and, when meningitis was present, material from this source was also examined bacteriologically. In a few instances, when I was unable to be present at the post-mortem examination, e.g., Cases 10 and 16, the lungs and certain other organs were sent to me at the laboratory from the hospital. All the autopsies were made within 24 hours of death.

Adopting this method of examination forty cases of broncho-pneumonia were examined during a period extending from 25th February, 1926, to the 19th February, 1929. Some of the main features of this investigation are detailed in the Appendix.

\section{BACTERIOLOGICAL RESULTS.}

In 33 out of the 40 cases a pure culture of Pfeiffer's bacillus was obtained from one source or another. In these cases it was possible to apply certain bacteriological tests for the identification of the strains isolated. The most important criterion for identifying Pfeiffer's bacillus is the fact that this organism grows on a special medium containing blood which has been modified by heat, and that it fails to grow on ordinary nutrient agar. Certain other tests were also applied such as ability to produce indol ; ability. to ferment glucose, galactose, xylose, fructose and in certain cases maltose with the production of acid but no gas. Certain agglutination reactions were also carried through. It is unnecessary here to enter into the details of these findings.

In five other cases, for one reason or another, a pure culture of Pfeiffer's bacillus was not obtained, though these organisms were recognized to be presen $t$ by microscopical examination. In Case 1, for example, a suitable medium was not available for the cultivation of the organism ; in Cases 4 and 19, owing to the abundant development of other organisms on the culture medium, it 
was not possible to isolate the bacillus in pure culture, and in Cases 21 and 29 owing either to some defect in the cultivating medium, or possibly to some error in the technique of preparing the cultures from the lungs, both specimens were sterile. The culture from the sputum in Case 21 was also sterile. In Case 29, no plates being a vailable, a series of agar slopes were employed and this in some measure explained the failure to isolate Pfeiffer's bacillus, although the organism was seen to be abundantly presen: in slides examined by the microscope. In these cases, if a microscopical examination can be relied on for identification, (admittedly a feeble method but of some value in experienced hands), Pfeiffer's bacillus was recognized. If these five cases are included with the 33 first mentioned then Pfeiffer's bacillus was demonstrated in 38 out of the 40 cases examined, i.e., 95 per cent.

In two cases only out of the 40 (No. 20 and 23) no evidence was obtained that Pfeiffer's bacillus played any part in the cause of the condition. They were cases of extensive suppuration of the lungs and in this respect they resembled Case 38. In this latter case, however, a culture was made from an enlarged gland at the hilum of the lung and here Pfeiffer's bacillus was still to be found, although in all other situations it had been completely replaced by a vast number of all sorts of bacteria. Possibly, if a lymphatic gland had been similarly examined in Cases 20 and 23, Pfeiffer's bacillus might have been revealed.

\section{Discission.}

The statement that Pfeiffer's bacillus was found in 95 per cent. of the cases examined is in itself of little value in deciding whether this organism is the cause of broncho-pneumonia in children; other facts must be taken into consideration. Among the most important of these is the fact that in early and acute cases (for example, Cases 3, 5, 9, 12. 14 22, 26, 31, 34, 35, 36, 39) the lung-puncture culture gave an almost pure growth of Pfeiffer's bacillus. In the sputum culture of these cases colonies of Pfeiffer's bacillus were numerous, but colonies of other organisms were also present, the latter being particularly numerous in Cases 35 and 36. Where the final issue had been still further prolonged, or where secondary infection by a particularly virulent organism had occurred, e.g., Cases 6, 8, 13, 18, 24, the lung-puncture culture showed the presence of other organisms in addition to Pfeiffer's bacillus, and in the same cases the sputum cultures showed that these other crganisms were present in still larger numbers. In cases which had progressed to a further stage of development, Cases 10 and 15, for example, colonies of Pfeiffer's bacillus were now scarcely to be found in the sputum cultures, although more numerous in the lung-puncture cultures. In certain more advanced cases, No. 4, 20, 23, 38 , where secondary invasion by all kinds of bacteria, but especially streptococci, had brought about the formation of multiple abscesses, Pfeiffer's bacillus could not be isolated from either the sputum or lung cultures. In Case 38 however, a few colonies of Pfeiffer's bacillus were obtained in a culture made from an enlarged lymphatic gland at the hilum of the lung. No culture from a similar source was made in the other cases of this group.

A careful and critical consideration of the evidence obtained during the examination of this series of 40 cases (some of this evidence has been detailed 
above but other observations more difficult to describe and record have been omitted), leaves little doubt in my mind that Pfeiffer's bacillus is the primary agent in causing the type of broncho-pneumonia which we have studied. This organism appeared to upset the normal resistance of the tissues of the respiratory tract against invasion by bacteria, so that organisms, which are found in limited numbers and strictly confined to the mucous surfaces of the respiratory tract in healthy persons, multiply greatly, invade and destroy the tissues, causing the development of abscesses in the lungs, or extending into the serous sacs cause suppuration there, or invading the blood give rise to a septicæmia or more rarely pyæmia.

Before referring to these organisms of secondary importance a word or two may be said about Pfeiffer's bacillus itself. This organism generally exercises its harmful effects locally, but occasionally it may gain access to the blood directly or through the lymphatic channels. It was present in the heart blood in pure culture in one case only, No. 3, but it was present in this situation with other organisms in five other cases. In Cases 11 and 12 it was present in the circulating blood together with streptococci ; in Case 9 with a coliform organism and staphylococci ; in Case 25 with a coliform organism and pneumococci, and in Case 28 together with two distinct types of pneumococcus. It was also present in pure culture in the cerebro-spinal fluid in Case 9, and was recovered from this situation before death. It was isolated in pure culture from the recent adhesions in the pleural cavity of Case 3, and from the pus in the pleural cavity together with pneumococci in Case 12. It was probably present, though not isolated in pure culture, together with pneumococci and streptococci in the pus trom the pleural cavity of Case 15. It was recovered from the pericardial fluid where it was present with pneumococci in Case 22 . Without doubt this organism spreads along the lymphatic channels, for out of three examinations it was recovered twice from the enlarged glands at the hilum of the lungs, i.e., in Cases 38 and 40.

Referring next to the secondary invading organisms, a streptococcus was most frequently found in the heart's blood. This organism was isolated in pure culture from this source in six cases, No. 4, 6, 7, 32, 33 and 36, and was present in two other cases, No. 11 and 12, together with Pfeiffer's bacillus. Streptococci were sometimes very abundantly present in the lungs, especially in cases where suppuration and abscess formation had developed, e.g., Cases 4, 20 , and 23. Streptococci were occasionally met with in the serous cavities; for example, in the peritoneum in pure culture in Case 30, and in the pleura, with other organisms, in Case 15.

Pneumococci were more rarely found in the blood. 'This organism was recovered in pure culture in this situation in Case 8 , and was present with Pfeiffer's bacillus in two other cases, No. 25 and 28 . In the latter case two distinct types of pneumococcus were present in the blood at the same time.

Some type of pneumococcus was most often found in cases which developed empyema, e.g., No. 22, 28 and 30. It was also present in the meninges in Case 22.

The most striking instance of secondary infection was found in Case 30 where a streptococcus was present in pure culture in the peritoneum, and a 
pneumococcus in the pleura, while Pfeiffer's bacillus was obtained in the lung-puncture culture almost uncontaminated with other organisms.

The question may be raised how far these cases of broncho-pneumonia are related to influenza. It is certain that the cases occurred in definite groups. As many as ten cases occurred in the months of February and March 1926 ; then a series of scattered cases, numbering in all eighteen, followed during the next eight months; another series of nine cases was examined in one month. It was definitely proved that the last group of cases occurred during a well marked epidemic of influenza in the city. Be this as it may, the bacteriological findings are just those met with in cases of influenzal broncho-pneumonia in adults, and if this fatal broncho-pneumonia of children is a manifestation of influenza, as I believe it is, then, coincidently with the appearance of cases of broncho-pneumonia, there should occur cases of capillary bronchitis, bronchitis, laryngitis and tonsillitis, diseases brought about by the same infection, but with desreasing degrees of extension of the virus along the respiratory tract in the order mentioned.

The question of the cause of influenza and its relation to Pfeiffer's bacillus has been well stated by Professor Muir² so long ago as 1919 when he wrote* :

"The question comes to be whether B. influenzæ (i.e. Pfeiffer's bacillus) is the essential cause of the disease or whether it is merely associated with an undiscovered virus, possibly ultra microscpoic as some have supposed. Microscopical examination of sections show that the influenza bacillus is specially related to the broncho-pneumonia, a special feature of influenza, it is often the predominant organism and may abound not only in the bronchioles but in the air vesicles beyond. In other words a lesion, common in influenza, and rare apart from it, is associated with the appearance of this delicate organism in large numbers and this lesion represents the farthest extension of bacterial growth in a downward direction; it seems not unlikely that B. influenzæ is the agent which so markedly lowers the general resistance of the bronchial passages. Thus it may be the real cause of the disease. On the other hand there is the possibility that the presence of an ultra-microscopic organism underlies all the phenomena and the results of further investigation on this point must be awaited. But in any case it appears to us that Pfeiffer's bacillus plays a very important part in the preduction of the characteristic lesions."

This is neither the time nor the place to enter into a discussion on this subject which must be deferred to a more suitable occasion.

\section{ConcLusions.}

Two general conclusions may be drawn from this investigation, First, that Pfeiffer's bacillus ( $B$. influenzo) is intimately associated with the bronchopneumonia of children; and secondly, that this organism opens a path to the invasion of the body by secondary organisms, particularly streptococci, pneumococci, and staphylococci, as well as other organisms. The latter organisms may be found in the blood, causing septicæmia or pyæmia, in the serous cavities giving rise to empyema, peritonitis, or pericarditis ; and they are frequently the cause of abscess formation in the lungs.

\section{REFERENCES.}

1. Liston, W.G., Indian J. Med. Res., Calcutta, VI, 418.

2. Muir \& Wilson, Brit. Med. J., Lond., 1919, i, 3. 


\title{
APPENDIX.
}

\author{
Notes on Cases Investigated.
}

Case. 1. K. G., aged 2 years, 4 months, died 25.2.26, after illness of 4 days. P.M. diagnosis, broncho-pneumonia with some confluence; Pfeiffer's bacillus microscopically in the sputum. No special media available for culture.

Case 2. A. A., aged 7 months, died 25.2.26, after illness of 8 days. P.M. diagnosis, confluent broncho-pneumonia ; Pfeiffer's bacillus on blood agar from the lung and microscopically abundantly present in sputum and lung films.

Case 3. J. F., aged 1 year, died 6.3.26, after illness of 12 days. P.M. diagnosis, bronchopneumonia with early confluence; a pure growth of Pfeiffer's bacillus from the heart, that organism also recovered from the lung and recent pleural adhesions.

Case 4. D. F., aged 1 month, died 8.3.26, after 1 day of apparent illness. P.M. diagnosis broncho-pneumonia of fulminating hæmorrhagic type; streptococcus from the heart, in pure culture. Numerous streptococci from lung abscess. Microscopically sputum showed numerous streptococci and a few gram negative bacilli, like Pfeiffer's bacillus.

Case 5. T. K., aged 1 year, died 8.3.26, after illness of 7 days. P.M. diagnosis, bronchopneumonia confluent in the lower lobes; Pfeiffer's bacillus in pure culture from lung; heart blood sterile.

Case 6. M. D., aged 1 year, 4 months, died 12.3.26, after 26 days illness. P.M. diagnosis, broncho-pneumonia with bronchiectasis; streptococcus pure culture from heart, lung culture showed streptococci, Pfeiffer's bacillus, and diphtheria, the latter was proved to be virulent.

Case 7. R. M., aged 2 years, 4 months, died 15.3.26, after 35 days illness. P.M. diagnosis, broncho-pneumonia with abscess formation; Pfeiffer's bacillus from abscess, streptococci from heart, sputum contained diphtheria proved to be virulent.

Case 8. H. M., aged 1 year, 1 month, died 18.3.26, after 17 days illness. P.M. diagnosis, confluent broncho-pneumonia with early bronchiectasis; a pure culture of pneumococcus from the heart, Pfeiffer's bacillus, pneumococcus, and micrococcus catarrhalis from the lung and sputum.

Case 9. T. P., aged 9 months, died 26.3.26, after 12 days illness. P.M. diagnosis, bronchopneumonia with meningitis. Pfeiffer's bacillus was recovered in a pure culture from the cerebrospinal fluid during life; a mixed culture of Pfeiffer's bacillus, staphylococci and a coliform organism from the heart ; lung culture almost pure Pfeiffer's bacillus.

Case 1.0. M. L., aged 6 years, died 31.3.26, after 8 days illness. P.M. diagnosis, old bronchiectasis with recent broncho-pneumonia; lungs sent to the laboratory where pus from abscess was found to contain many streptococci, a few Pfeiffer's bacilli, lung cultures showed some Pfeiffer's bacilli, many streptococci, staphylococci, pneumococci and other otganisms.

Case 11. C. B., aged 5 years, died 5.11.26, after 13 days illness. P.M. diagnosis, empyema with welldefined abscesses at base of lungs; a mixed culture of Pfeiffer's bacillus with streptococci from the heart; lung cultures showed many streptococci, some Gram-negative bacilli, and M. catarrhalis.

Case 12. G. M., aged 2 years, 6 months, died 10.11.26, after 12 days illness. P.M. dicunosis, mixed broncho- and lobar pneumonia; pleural fluid contained many pneumococci but some Pfeiffer's bacilli ; lung culture, practically pure Pfeiffer's bacilli ; sputum, a mixed culture of Pfeiffer's bacilli and streptococci.

Case 13. M. D., aged 10 months, died 18.11.26, after 22 days illness. P.M. diagnosis, lobar pneumonia; Pfeiffer's bacillus, pneumococci and catarrhalis from lung culture ; heart culture sterile.

Case 14. A. C., aged 2 months, died 1.12.26, after 10 days illness. P.M. diagnosis, bronchopneumonia with extensive collapse. Pfeiffer's bacillus from throat before death, pure culture of Pfeiffer's bacillus from lung, heart sterile.

Case 15. J. S., aged 1 year and 3 months, died 1.12.26, after 75 days illness from long continued broncho-pneumonia with bronchiectasis and empyema, pus from pleura showed Gram negative bacilli with pneumococci and streptococci, a few colonies of Pfeiffer's bacillus from the sputum. Heart blood sterile. 
Case 16. J. K., aged 1 year, 1 month, died 13.12.26, after 14 days illness from bronchopneumonia with moderate confluence and some dilatation of the bronchi; organs sent to the laboratory, where from the lung culture six colonies of Pfeiffer's bacillus were recovered; pneumococci and streptococci were isolated from the spleen.

Case 17. R. A., aged 3 years, 7 months, died 14.12.26, after 7 days illness, This was a case of broncho-pneumonia following measles; a rich culture of Pfeiffer's bacillus was obtained from the sputum. Heart culture sterile.

Case 18. G. M., aged 4 years, died 18.2.27, after 15 days illness from broncho-pneumonia with irregular confluence; Pfeiffer's bacillus and streptococci from the lung, these organisms together with diphtheria were isolated from the suptum; heart culture sterile.

Case 19. T. C., aged 1 year and 5 months, died 28.2.27, after 45 days illness, from pulmonary tuberculosis with super-added acute pneumonia; sputum showed abundant colonies of a Gram negative bacillus with numerous colonies of pneumococci and streptococci. Pfeiffer's bacillus, however, was not isolated in pure culture ; heart blood culture sterile.

Case 20. J. M., aged 1 year and 4 months, died 3.5.27, after an illness of 25 days. This was a typical lobar pneumonia with suppuration; streptococci, catarrhalis and pneumococci together with a Gram negative bacillus (which was not Pfeiffer's bacillus) were isolated from the cultures in this case ; no evidence of the presence of Pfeiffer's bacillus was obtained ; heart blood culture was sterile.

Case 21. M. R., aged 8 months, died 21.3.27, after 37 days illness from confluent bronchopneumonia with empyema; numerous bacilli morphologically resembling Pfeiffer's bacillus were present in the sputum, the cultures however were sterile; heart blood culture sterile.

Case 22. G. T., aged 1 year, died 22.3.27, after 16 days illness from broncho-pneumonia with pericarditis, empyema, and cerebro-spinal meningitis; cultures from the sputum gave an abundant growth of Pfeiffer's bacillus; cultures from the lungs and cerebro-spinal fluid were sterile; the pericardial fluid gave a mixed culture of Pfeiffer's bacillus with pneumococcus; heart culture was sterile.

Case 23. B. H., aged 5 months, died 24.3.27, after 10 days illness from confluent bronchpneumonia ; the bacterial feature of the sputum viewed microscopically was the large number of streptococci present ; cultures from the sputum gave streptococci, staphylococci, together with a diphtheria-like bacillus. Similar organisms in the lung culture, no Pfeiffer's bacilli seen ; heart culture sterile.

Case 24. J. C., aged 9 months, died 24.3.27, after 5 days illness from confluent bronchopneumonia; sputum showed many colonies of Pfeiffer's bacillus with some staphylococci and pneumococei ; heart culture sterile.

Case 25. M. L., aged 1 year, died 27.3.27, after 35 days illness from confluent bronchopneumonia with empyema; the lung culture in this case gave an almost pure growth of Pfeiffer's bacillus ; a mixed culture of Pfeiffer's bacillus, a type of pneumococcus, and a coliform bacillus was obtained from the heart.

Case 26. A. D., aged 2 years 3 months, died on 5.4.27, after 10 days illness from discrete broncho-pneumonia; a practically pure culture of Pfeiffer's bacillus was obtained from the lung ; the sputum showed many colonies of Pfeiffer's bacillus but the heart blood was sterile.

Case 27. R. P., aged 1 year, 5 months, died 7.5.27, after 8 days illness from bronchopneumonia with some confluence; the lung culture showed many colonies of Pfeiffer's bacillus with catarrhalis and staphylocei ; the sputum culture also showed many colonies of Pfeiffer's bacillus.

Case 28. G. S., aged 7 months, died $\mathbf{6 . 6 . 2 7}$, after 21 days illness from a typical lobar pneumonia with empyema; the pleural fluid gave a pure growth of pneumococcus; the sputum culture showed many colonies of Pfeiffer's bacillus; the lung culture also showed many colonies of Pfeiffer's bacillus but some colonies of pneumococcus and catarrhalis were also present ; the heart culture was a mixed growth of Pfeiffer's bacillus with two distinct types of pneumococci.

Case 29. W. T., aged 5 months, died 31.10.27, after 3 days illness from broncho-pneumonia with acute peritonitis ; the culture from the heart was sterile, so also was the culture from the lung; the peritoneal exudate showed many streptococci ; in the sputum many minute Gram negative bacilli like Pfeiffer's bacillus were seen microscopically but no culture of that organism was recovered, 
Case 30. A. A., aged 1 year, 3 months, died 24.1.28, after 40 days illness from bronchopneumonia and empyema; the peritoneal exudate gave a pure culture of streptococci; the pleural fluid contained typical pneumococci while the culture from the lung gave an almost pure growth of Pfeiffer's bacillus.

Case 31. D. L., aged 1 year, 6 months, died 14.11.28, after 8 days illness from confluent broncho-pneumonia ; almost a pure culture of Pfeiffer's bacillus was obtained from the lung and these organisms were abundant in the culture from the sputum; the heart culture was sterile.

Case 32. I. P., aged 5 months, died 5.2.29, after 21 days illness from partially confluent broncho-pneumonia ; sputum culture showed many streptococci and some colonies of Pfeiffer's bacillus ; the lung culture gave an almost pure growth of Pfeiffer's bacillus; the heart culture gave a pure growth of a streptococcus.

Case 33. I. M., aged 9 months, died 5.2.29, after two days illness. This child died after a fit. A small patch of pneumonia was found in one lung; the sputum culture showed many streptococci and catarrhalis but some Pfeiffer's bacillus colonies were also obtained; the lung culture was almost pure Pfeiffer's bacillus, the heart culture gave a pure streptococcus.

Case 34. R. B., aged 2 years, died 7.2.29, after 30 days illness from discrete bronchopneumonia with empyema; the lung culture gave pure Pfeiffer's bacillus ; the pus from the pleura gave a pure growth of a type of pneumococcus; the heart culture was sterile.

Case 35. R. R., aged 3 years, died 8.2.29, after 14 days illness from broncho-pneumonia; the lung culture showed a pure growth of Pfeiffer's bacillus ; the sputum culture contained many colonies of that bacillus with other organisms, the heart culture was sterile.

Case 36. A. G., 1 year, 2 months, died 11.2.29, after 10 days illness from discrete bronchopneumonia ; the long culture was almost pure Pfeiffer's bacillus and the sputum culture showed a few colonies of that bacillus with many streptococci and other organisms, the heart culture was sterile.

Case 37. J. A., aged 2 years 4 months, died 11.2.29, after 7 days illness from tubercular meningitis with broncho-pneumonia ; colonies of Pfeiffer's bacillus were abundant in the sputum culture. No other cultures were made as the organs had been removed before $\mathrm{I}$ arrived at the autopsy.

Case 38. J. S., aged 5 years, died 12.2.29, after 18 days illness from broncho-pneumonia with multiple abscess formation ; the abscesses were so numerous that a lung puncture could not be made without entering a small abscess cavity; the sputum swarmed with organisms. No Pfeiffer's bacilli were recovered from the usual sources but in this case a culture was made from a gland at the hilum of the lung, from this a few colonies of Pfeiffer's bacillus were obtained with colonies of other organisms.

Case 39. S. M., aged 2 years, 11 months, died 19.2.29, after 14 days illness from bronchopneumonia ; an almost pure culture of Pfeiffer's bacillus was obtained from the lung; the sputum culture showed an abundant growth of that organism. A culture from a lymph gland at the hilum of the lung and from the heart were sterile.

Case 40. J. R., aged 8 months, died 19.2.29, after 11 days illness from broncho-pneumonia which was confluent in one lung and early in the other ; numerous colonies of Pfeiffer's bacillus were obtained in the lung culture mixed with other organisms; a few colonies of Pfeiffer's bacillus were also found with some other organisms in a culture made from a lymph gland in the lung. 